УДК 539.3, 621.891

\title{
The mechanism of movement in a medium of the intermediate layer between the contacting bodies
}

\author{
R.V. Goldstein ${ }^{\dagger}$, N.M. Osipenko \\ †goldst@ipm.net.ru
}

Institute for Problems in Mechanics RAS, prospect Vernadskogo, 101-1, Moscow 119526, Russia

Friction conditions at the contact of bodies are connected with the structure of the contact area, in particular, with occurring the products of their destruction or severe deformation in this area such that further interaction of bodies takes place through the elements of the structure of an intermediate layer. A mechanism of internal motions in the intermediate layer which can alter its structure at friction is demonstrated and analyzed in the paper. The conditions and basic mechanism adjusting the formation of the structure at the interface at friction are determined. The interaction between the bodies is carried out by rolling between the structural elements of the intermediate layer (balls). Loads from contacting bodies lead to the convergence of balls with increasing total tangential displacements. This process is represented as an analog of the transformed (viscous) friction, whereby when the body is moved with friction on the surface under the action of some force then the appearance of an additional force of another direction is also accompanied by the displacement of the body in the direction of this force no matter how small it may be. This approach can be extended for an analysis of a system of blocks in the intermediate layer if their relative motion occurs in the sliding regime. An analytical model of convergence of the rolling elements at the interface under shear with compression on the basis of the laws of the transformed (viscous) friction was suggested. The model was confirmed experimentally. It is shown that the rate of convergence does not depend on the direction of the main drawing together of contacting bodies. The speed of convergence increases with the decreasing the distance between the balls until they touch. The described process represents a basis for the scenario of the particles grouping in the intermediate layer and aligning them into compact formations. Accounting for this phenomenon can be useful for technological processes such as chemicalmechanical polishing. Mutual influence and motion of abrasive particles, formation of their conglomerates can change the intensity of their action on the treated surface.

Keywords: friction, intermediate layer, stress, strain, structure.

\section{Механизм движений в среде промежуточного слоя между контактирующими телами}

\author{
Гольдштейн Р.В. ${ }^{\dagger}$, Осипенко Н.М. \\ †goldst@ipm.net.ru
}

Институт проблем механики РАН, проспект Вернадского 101-1, 119526, Москва, Россия

Условия трения на контакте тел связаны со структурой области контакта - появлением в ней продуктов разрушения или интенсивной деформации, и дальнейшее взаимодействие тел осуществляется через элементы структуры промежуточного слоя. Продемонстрирован механизм внутренних движений, способных видоизменять структуру в среде промежуточного слоя между контактирующими телами при трении. В работе определены условия и базовый механизм, управляющие формированием структуры на интерфейсе при трении, когда взаимодействие между телами осуществляется посредством перекатывания между ними структурных элементов промежуточного слоя (шариков). Нагрузки со стороны контактирующих тел приводят к сближению шариков по мере увеличения суммарных касательных смещений. Этот процесс представлен как аналог преобразованного (вязкого) трения, согласно которому если тело перемещается под действием некоторой силы в режиме трения по поверхности, то появление дополнительной силы другого направления, в частности силы $T$, приводит к перемещению тела и в направлении действия этой силы, как бы мала она ни была. Такой подход может быть распространен и на систему блоков в промежуточном слое, взаимное перемещение которых происходит в режиме скольжения. Построена аналитическая модель сближения элементов качения на границе контакта в условиях сдвига со сжатием на основе закономерностей преобразо- 
ванного (вязкого) трения. Модельный подход подтвержден экспериментально. Показано, что темп сближения не зависит от направления основного смещения контактирующих тел. Скорость сближения возрастает по мере сокращения расстояния между шариками вплоть до их соприкосновения. Описанный процесс лежит в основе сценария группировки частиц в промежуточном слое и выстраивании их в компактные образования. Учет этого явления может быть полезен для технологических процессов, таких как химико-механическое полирование. Взаимное влияние и перемещение частиц абразива и образуемые ими конгломераты могут менять интенсивность их воздействия на обрабатываемую поверхность.

Ключевые слова: трение, промежуточный слой, напряжение, перемещение, структура.

\section{1. Введение}

В условиях силового контакта, сопровождаемого локальным разрушением контактирующих тел, изменение эффективных условий трения при движении тел может быть связано с изменением структуры области контакта - появлением в ней продуктов разрушения или интенсивной деформации, во многих случаях облегчающих движение.

В результате дальнейшее взаимодействие тел осуществляется через элементы структуры промежуточного слоя. Некоторые модели таких явлений обсуждались в предыдущих работах авторов [1-4]. О правомерности этой постановки задачи свидетельствуют многочисленные экспериментальные данные [5]. Упорядоченное разрушение на границе контакта при сдвиге может быть одним из главных механизмов износа контактирующих поверхностей. Ранее [2] при рассмотрении сценариев разрушения на границе контакта был выделен этап инициирования промежуточного слоя (третьего тела). Его эволюция и роль в механизме трения и износа зависят от природы материала и условий контакта. В связи с этим можно выделить несколько ведущих типов третьего тела, как это сделано, например, в [3].

Ниже определены условия и базовый механизм, управляющие формированием структуры на интерфейсе при трении в ситуации, соответствующей варианту рис.1b. В этом варианте два упругих тела, имеющие плоский шероховатый контакт, подвержены в точках, удаленных от области контакта, однородному взаимному сжатию $\left(\sigma_{\infty}\right)$ и сдвигу $\left(\tau_{\infty}\right)$. Взаимодействие между телами в области проскальзывания осуществляется посредством перекатывания между ними структурных элементов промежуточного слоя, образующихся в результате разрушения приповерхностных слоев трущихся тел [4], либо внесенных извне. К последним могут быть отнесены, например, частицы абразива.
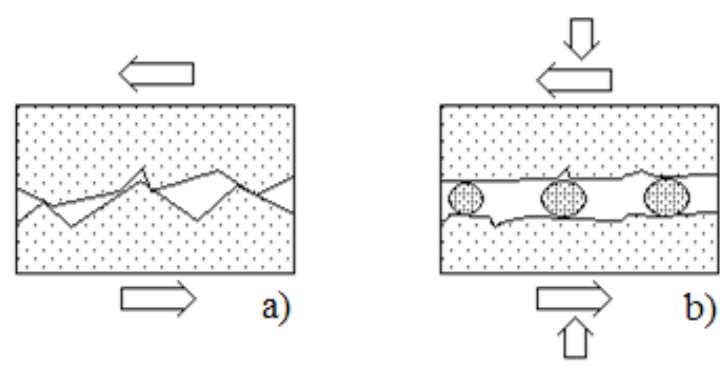

Проведено экспериментальное моделирование процесса и построена аналитическая модель перекатывания элементов на границе контакта в условиях сдвига со сжатием.

\section{2. Сценарий движения частиц в промежуточном слое}

Контактному взаимодействию с трением и поверхностным разрушением присущи механизмы саморегуляции, в результате которых происходит развитие структуры промежуточного слоя. Ниже рассмотрен один из механизмов такой саморегуляции, основанный на возможности перемещения частиц в промежуточном слое под действием малых возмущений напряженного состояния, создаваемых в окрестности частиц. В стационарном режиме существования структур трения, предпочтительность того или иного состояния связывается с величинами полной потенциальной энергии упругой системы, соответствующими этим состояниям. Из возможных состояний более вероятно такое, в котором полная потенциальная энергия системы меньше. Характер взаимодействия тел во внешней задаче при этом зависит от взаимного расположения элементов в промежуточном слое. Механизм регулирования взаимного расположения частиц в рамках внутренней задачи промежуточного слоя является предметом внимания в данной работе.

При силовом контакте тел частицы в промежуточном слое исполняют роль штампов по отношению к контактирующим через них телам. Анализ напряженного состояния в окрестностях штампов для различных ситуаций внешних нагрузок, формы и взаимного расположения можно найти в многочисленных публикациях [6 - 10 и др.]. Обзор работ по механике дискретного контакта содержится, например, в [11].
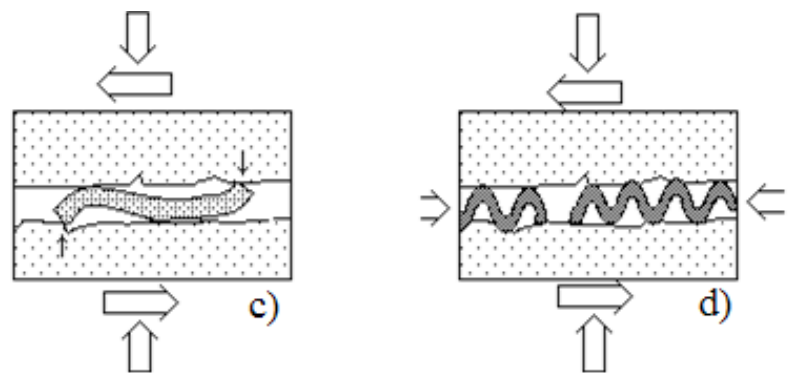

Рис. 1. Варианты третьего тела, формирующиеся по мере возрастания напряжений сжатия на контакте [3].

Fig. 1. Variants of the third body, formed with increasing compressive stresses at the contact [3]. 
Рассмотрим пример элементарного акта взаимодействия, поясняющий качественную картину процесса. Для простоты считается, что упругие свойства контактирующих тел одинаковы, а одинаковые твердые частицы имеют сферическую форму.

Пусть на некотором участке промежуточного слоя на плоской границе упругого тела расположены два жестких шарика радиуса $\mathrm{R}$, расстояние между которыми $\mathrm{S}$ (рис.2).

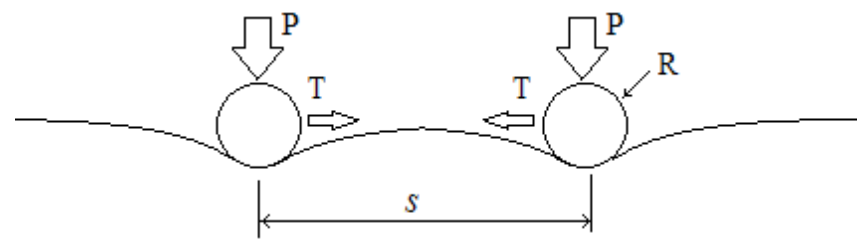

Pис. 2. Схема взаимодействия близко расположенных шариков. Fig. 2. Scheme of interaction of closely spaced beads.

Под действием приложенных нормальных нагрузок $P$ шарики вдавливаются в поверхность тела. Вследствие конечности расстояния между ними появляются малые силы $T$, направленные навстречу друг другу, что характерно для близко расположенных штампов различной формы [10]. При статической нагрузке это, как правило, не приводит к перемещению штампов. Ситуация меняется, если привести шарики в движение с некоторой скоростью вдоль поверхности контакта (например посредством перемещения жесткой плиты, передающей на шарики нагрузку $P$ ). В этом случае возможна реализация эффекта преобразованного трения $[12,13]$, согласно которому, если тело перемещается под действием фиксированной силы в режиме трения по некоторой поверхности, то появление дополнительной силы другого направления, в частности силы $T$, приводит к перемещению тела и в направлении действия этой силы, как бы мала она ни была. В этом режиме нет принципиального отличия в форме записи для сухого трения и трения качения.

Приведем необходимые оценки. Оценим силу $T$, полагая, что шарики внедряются в упругое полупространство как жесткие сферы. Напомним соотношения для внедрения одиночного шарика (задача Герца) [14].

Вертикальные смещения точек нагруженной области

$$
u_{z}=\frac{1-v^{2}}{E} \frac{\pi p_{0}}{4 a}\left(2 a^{2}-r^{2}\right) ; \quad r \leq a,
$$

где $a$ - радиус области контакта, $p_{0}$ - среднее давление.

Сближение удаленных точек

$$
\delta=\frac{\pi p_{0} a}{2 E}=\left(\frac{9 P^{2}}{16 R E^{2}}\right)^{1 / 3}
$$

Радиус области контакта контакта и среднее давление

$$
a=\frac{\pi p_{0} R}{2 E}=\left(\frac{3 P R}{4 E}\right)^{1 / 3} ; \quad p_{0}=\left(\frac{6 P E^{2}}{\pi^{3} R^{2}}\right)^{1 / 3}
$$

Вертикальные и горизонтальные смещения поверхности под действием сосредоточенной силы

$$
u_{z}=\frac{1-v}{2 \pi G} \frac{P}{r}=\frac{1-v^{2}}{\pi E} \frac{P}{r} \quad u_{r}=-\frac{1-2 v}{2 \pi G} \frac{P}{r}
$$

Сопоставим эти решения в части вертикальных смещения их на границе области контакта $(r=a)$ и на некотором удалении от сосредоточенной силы $r^{*}$.

$$
\begin{aligned}
& \frac{1-v^{2}}{\pi E} \frac{P}{r^{*}}=\frac{1-v^{2}}{E} \frac{\pi p_{0} a}{4} \\
& r^{*}=\frac{4}{\pi}\left(\frac{2 P R}{9 E}\right)^{1 / 3} ; \frac{r^{*}}{a}=\frac{8}{3 \pi} \approx 0.85
\end{aligned}
$$

Таким образом, для удаленных точек поверхности $(r>a)$ вертикальные смещения поверхности от сосредоточенной силы, близки к таковым для внедрения жесткого шарика с немного меньшим размером пятна контакта (или соответственно меньшей величиной эффективного радиуса). Пренебрегая этой разницей, будем оценивать вертикальные смещения поверхности полупространства вдали от области внедрения шарика как смещения от сосредоточенной силы Р, равной вертикальной нагрузке на шарик.

На удалении $r$ от области приложения силы $P$ поверхность полупространства наклонена в сторону этой области. Приложенная там вертикальная сила может быть разложена на составляющие, в том числе направленную к области приложении силы $P$ силу T.

Производная от вертикального смещения под действием сосредоточенной силы

$$
u_{z}^{\prime}=-\frac{1-v^{2}}{\pi E} \frac{P}{r^{2}}
$$

Скатывающая сила в точке наклонной поверхности

$$
T=P u_{z}^{\prime}=-\frac{1-v^{2}}{\pi E} \frac{P^{2}}{r^{2}}
$$

Обратимся далее к процессу движения в режиме преобразованного сухого трения [12]. Приведем систему двух шариков в движение в направлении поперек линии, соединяющей их центры. Действие скатывающей силы на соседний шарик в паре равносильно появлению дополнительной силы в системе, что, как отмечено выше, приводит к смещению в направлении этой силы. Сопротивление трения в дополнительном движении в направлении поперек основного перемещения определяется соотношением

$$
F_{1}=-f P \frac{\dot{x}}{\sqrt{\dot{x}^{2}+\dot{y}^{2}}}
$$

где $x, y$ координаты, выбранные таким образом, что основное движение направлено по координате $y, f$ - коэффициент трения.

$$
\begin{aligned}
& \text { При } \dot{y}=\text { const }=\mathrm{v} ;|\dot{\mathrm{x}}|<<\mathrm{v} \\
& \qquad F_{1}=-b \dot{x} ; b=\frac{f P}{\mathrm{v}}=\text { const }
\end{aligned}
$$

(режим вязкого трения).

Путь в поперечном направлении

$$
\begin{aligned}
& x=\int_{0}^{t} \frac{F_{1}(t) \mathrm{v}}{f P} d t ; \\
& \frac{d x}{d t}=\frac{d x}{d y} \frac{d y}{d t}=\frac{d x}{d y} \mathrm{v} ; \\
& \mathrm{x}=\int_{0}^{y} \frac{F_{1}(y)}{f P} d y
\end{aligned}
$$


Распространим для пары взаимодействующих частиц - шариков принцип преобразованного трения на движение в любом направлении относительно направления основного перемещения системы, если речь идет о взаимном сближении или отдалении частиц. Иными словами, дополнительное взаимное перемещение взаимодействующих частиц в режиме преобразованного трения может происходить в любом направлении относительно направления основного движения, в том числе и в направлении этого движения. Приравнивая сопротивление трения скатывающей силе, получим

$$
-T=P f \frac{d s}{d y}
$$

где $s$ - расстояние между частицами.

На основе соотношений (7) и (11) запишем закон сближения частиц

$$
-\frac{1-v^{2}}{\pi E} \frac{P}{s^{2}}=f \frac{d s}{d y}
$$

Если в начальном положении характерное расстояние между частицами $r_{0}$, отсюда получим расстояние между частицами по мере развития основного движения

$$
s(L)=\left(r_{0}^{3}-3 \frac{1-v^{2}}{f \pi E} P L\right)^{1 / 3}
$$

где $L$ - путь основного движения пары взаимодействующих частиц.

На рис.3 приведен пример расчета сближения шариков применительно к ситуации проведенного и описанного в п. 3 эксперимента $(E=57.4 \kappa П а, P=9 H)$.

\section{3. Эксперимент}

Для демонстрации эффектов движения в промежуточном слое был проведен эксперимент.

В фиксированном промежутке между упругой податливой подложкой и жесткой подвижной пластиной, помещались стальные шарики (рис.4). Пластина получала

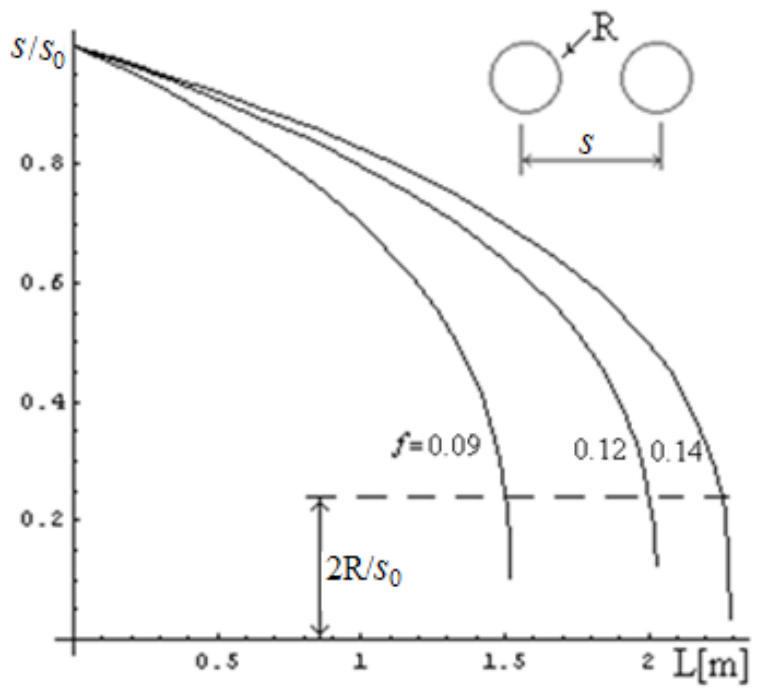

Рис. 3 Характер сближения двух шариков на упругой подложке. Fig. 3 The nature of convergence of two beads on an elastic substrate.

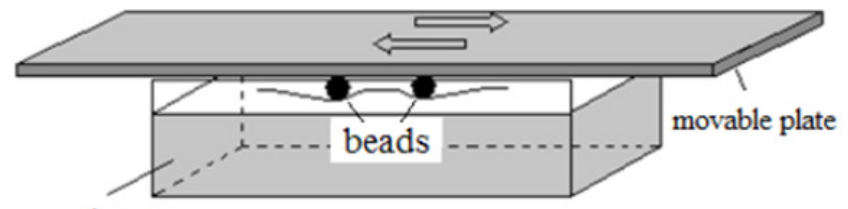

substrate

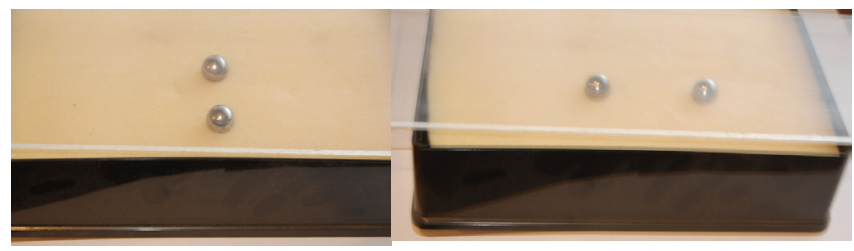

Рис. 4. Схема эксперимента. Внизу показаны варианты исходного размещения шариков между подвижной пластиной и подложкой.

Fig. 4. Scheme of the experiment. Variants of original location of beads between movable plate and substrate are shown below.

возвратно-поступательное движение. Проводились наблюдения за относительным смещением шариков при их сближении из первоначально разделенного состояния.

В эксперименте были использованы одинаковые стальные шарики радиусом 8мм и податливая упругая подложка толщиной 80мм, содержащая два слоя. Нижний поролоновый слой толщиной 70мм скреплен со слоем губчатой резины толщиной 10мм, который был непосредственным основанием для движения шариков. Подвижная жесткая пластина из ПММА, задававшая движение шариков, при каждом акте продольного возвратно-поступательного движения перемещалась на $10 \mathrm{~cm}$. Все контрольные эксперименты проводились при фиксированном вертикальном сближении верхней пластины и подложки 12мм.

Эффективная жесткость подложки, определенная по усилию при пробном внедрении шарика на глубину $12 \mathrm{Mм}, E=57.4 \kappa П а$.

Эффективный коэффициент трения качения шарика по подложке при глубине внедрения 12 мм, найденный в независимом испытании, был в диапазоне $f \sim 0.10-0.13$.

\section{4. Обсуждение результатов экспериментов}

Результаты измерений сближения шариков приведены на рис.5. Смыкание их происходит при $s=2 R$. Обращает внимание факт, что изменение расстояния между шариками в процессе сближения практически не зависит от исходной ориентации шариков относительно направления основного смещения.

Поскольку в проведенном эксперименте задано погружение системы шариков в основание по нормали к плоскости контакта на фиксированную глубину, в законе сближения (12),(13) нужно связать эффективную нагрузку Р с этим смещением. Вообще говоря, при этом нужно учитывать влияние прогиба под нагрузкой соседнего шарика. Тогда эффективное вдавливание для индивидуального шарика будет

$$
\delta^{*}=\delta-u_{z}(r)=\delta-\frac{1-v^{2}}{\pi E} \frac{P}{r}
$$


или

$$
\delta=\delta^{*}+u_{z}(r)=\left(\frac{9 P^{2}}{16 R E^{2}}\right)^{1 / 3}+\frac{1-v^{2}}{\pi E} \frac{P}{r}
$$

Получающиеся выражения для эффективной нагрузки $P$ громоздки. Поэтому для упрощения обратим внимание на характер функции $s(L)$ на рис.3. Можно видеть, что ее крутизна быстро возрастает только в непосредственной близости от точки смыкания шариков. Это связано с тем, что в (15) при $r>R$ первое слагаемое значительно больше второго. Поэтому, интересуясь оценкой влияния дальних взаимодействий, можем пренебречь изменением силы $P$ из-за прогиба подложки.

Из (2) получим

$$
P=\frac{4 E}{3} \sqrt{\delta^{3} R}
$$

Соответственно, закон сближения (13) принимает вид

$$
s(L)=\left(s_{0}^{3}-4 \frac{1-v^{2}}{f \pi} \sqrt{\delta^{3} R} L\right)^{1 / 3}
$$

На рис.6. показана эта функция, рассчитанная для условий эксперимента, в сочетании с его результатами. Можно видеть, что на расстояниях более $s \sim 30$ мм она хорошо описывает результаты эксперимента (при $f=0.12$ ).

В рассмотренной оценке не учитывалось изменение профиля поверхности подложки вследствие присутствия касательных сил, возникающих при движении шариков под нагрузкой и искажения при этом вектора сил

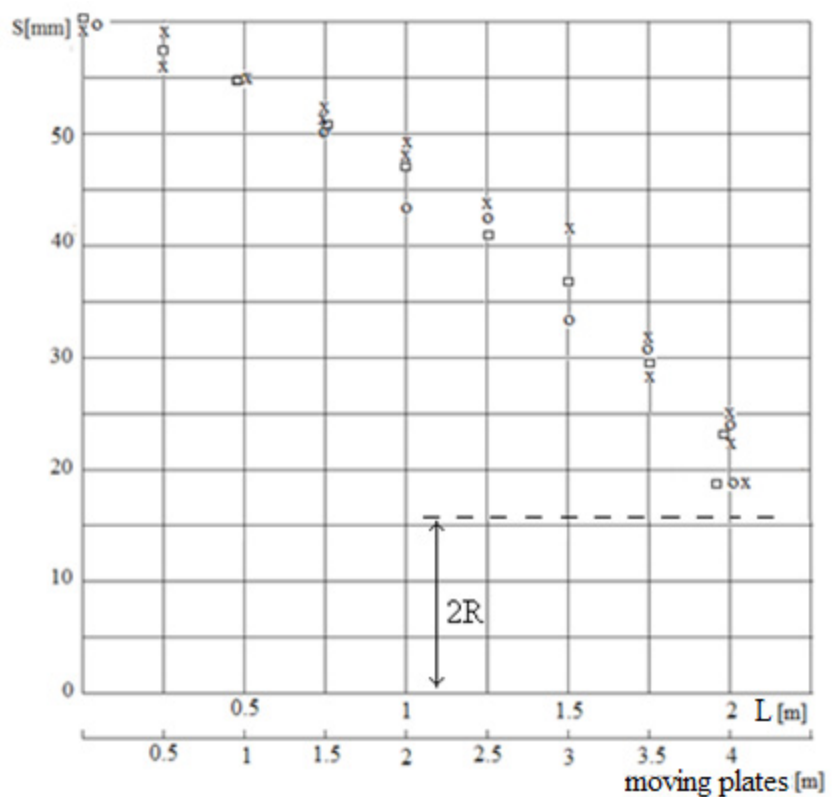

Рис. 5. Сближение шариков (эксперимент) $\times-$ шарики расположены друг за другом в направлении основного движения, о - шарики расположены на оси, наклоненной под углом $45^{\circ}$ к направлению движения, $\square-$ шарики расположены на оси поперек направления основного движения.

Fig. 5. Convergence of beads (experiment) $x$ - beads arranged one behind another in the direction of the main movement, $\mathrm{o}-$ beads arranged on an axis inclined at an angle of $45^{\circ}$ to the direction of movement, $\square-$ beads arranged on an axis transverse to the main direction of movement. взаимодействия шариков. Судя по результатам экспериментов, фиксирующих отсутствие влияния ориентации взаимного расположения шариков, это влияние на суммарный эффект сближения исчезающе мало.

Однако его кинематическое проявление можно обнаружить, анализируя периодические движения отдельного шарика в паре в процессе возвратно-поступательного перемещения, например для варианта расположения шариков вдоль направления основного движения. Схема движений в такой паре шариков показана на рис.7. Шарики на каждом шаге возвратно-поступательного движения сближаются поочередно. При поступательном движении такой кинематической системы в течение одного шага сближение происходит в основном за счет смещения одного шарика. Заметим, что в варианте исходного расположения пары шариков поперек направления основного движения их сближение происходит одновременно.

\section{5. Заключение}

Продемонстрирован механизм внутренних движений, способных видоизменять структуру в среде промежуточного слоя между контактирующими телами при трении. Показано, что в случае, когда исходные элементы промежуточного слоя представляют собой шаровидные блоки, способные перекатываться под действием касательных сил, нормальные нагрузки со стороны контактирующих тел приводят к сближению блоков по мере увеличения суммарных касательных смещений. Этот процесс может быть представлен как аналог преобразованного (вязкого) трения. Модельный подход подтвержден экспериментально. Он может быть распространен и на систему блоков, взаимное перемещение которых происходит в режиме скольжения. Темп сближения блоков не зависит от направления основного смещения

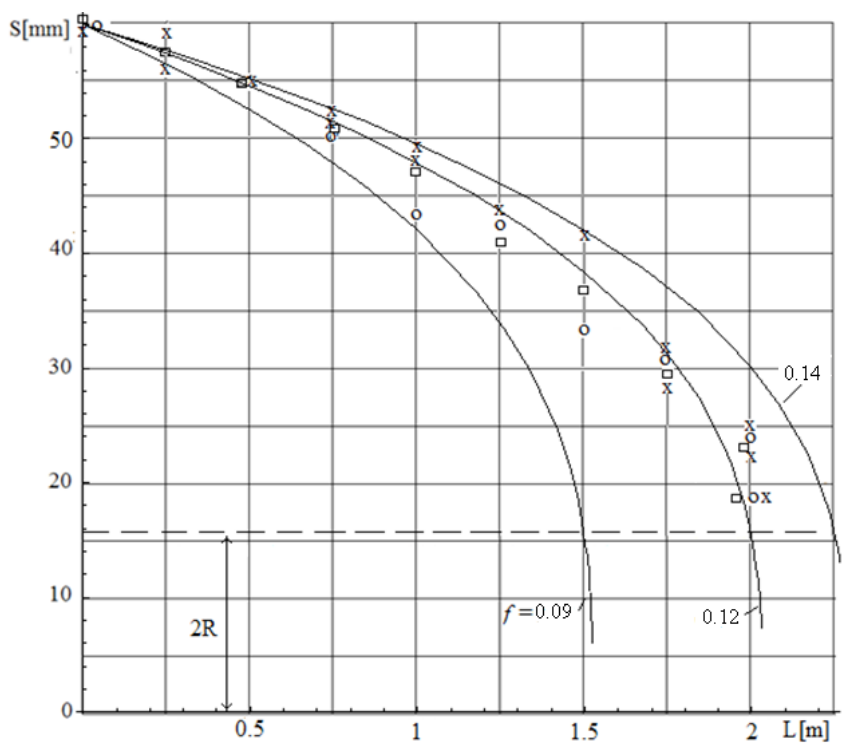

Рис. 6. Сравнение результатов эксперимента с теорией.

Fig. 6. Comparison of the experimental results with the theory. 


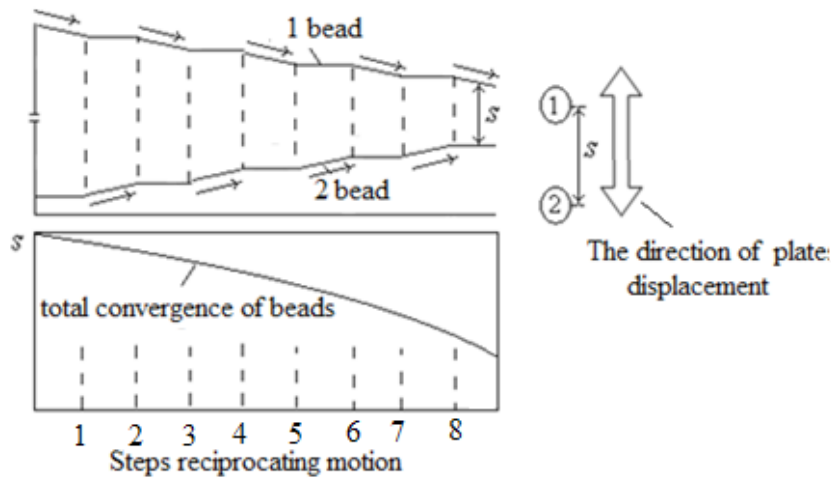

Рис. 7. Схема сближения шариков, расположенных друг за другом по направлению движения. Вверху - схема движения каждого шарика, внизу - суммарное сближение.

Fig. 7. Scheme of convergence of beads located next to each other in the direction of movement. Above - scheme of displacement of each bead, the bottom - total convergence.

контактирующих тел.

Описанный процесс лежит в основе сценария группировки частиц в промежуточном слое и выстраивании их в компактные образования.

Благодарность. Работа выполнена при поддержке Программы фундаментальных исследований Отделения энергетики, машиностроения, механики и процессов управления РАН №4.11.

Acknowledgements. This work was supported by the Program for the Basic Research 4.11 of the Department of Energetic, Machinery, Mechanics and Control Processes of the Russian Academy of Science.

\section{Литература / References}

1. N.M. Alekseev, R.V. Goldstein and N.M. Osipenko. 12(6), 965-973. (1991). (in Russian) [Н.М. Алексеев, P.В. Гольдштейн, Н.М. Осипенко. Трение и износ. 12(6), 965-973 (1991).]

2. N. M. Alekseev, R. V. Goldstein, and N. M. Osipenko, Mechanics of Solids, No. 5, 134-143 (1992). (in Russian). [Н.М. Алексеев, Р.В. Гольдштейн, Н.М. Осипенко. Изв. РАН. МТТ, 5, 134-143. (1992).]

3. R.V. Goldstein, N.M. Osipenko. Letters on materials.
2(3). 121 (2012). (in Russian) [Гольдштейн P.B., Осипенко Н.М. Письма о материалах. 2(3). 121(2012).]

4. R.V. Goldstein, N.M. Osipenko. Some mechanical models of chemical-mechanical polishing processes. In: Deformation and Fracture in Technological Processes. Key Engineering Materials. 528. 33-44, (2013). ISSN: 1662-9795.

5. E.A. Marchenko. About the nature of fracture of metal surfaces by friction. M : Nauka. (1979). 120p. (in Russian). [Е. А. Марченко. О природе разрушения поверхности металлов при трении. М.: Наука. (1979). 120c.]

6. I.V. Kragelsky. Friction and wear. M: Mashgiz. (1962), 384p. (in Russian). [И.В. Крагельский Трение и износ в машинах. М.: Машгиз. (1962), 384c.]

7. I.G. Goryacheva, M.N. Dobychin. Contact problems in tribology. M .: Machinery (1988). 256 p. (in Russian). [И.Г. Горячева, М.Н. Добычин. Контактные задачи в трибологии. М.: Машиностроение, (1988). 256c.]

8. A.E. Andreikiv, V.V. Panasiuk. DAN UkrSSR, A6. 26-32. (1971). (in Russian). [A.Е. Андрейкив, В.В. Панасюк. ДАН УкрССР, А.6. 26-32. (1971).]

9. I.G. Goryacheva. Mechanics of frictional interaction. M . Nauka. (2001) 478.p. (in Russian). [И.Г. Горячева Механика фрикционного взаимодействия. М.: Наука. (2001), 478c.]

10. I.I. Argatov. Advances in Mechanics. 4, 8-40. (2002). (in Russian) [И.И. Аргатов. Успехи механики. 4, 8-40. (2002).]

11. I.G. Goryacheva, O.G. Chekina. Mechanics of discrete contact. In: Mechanics of Contact Interactions. M 418437. Nauka, Moscow. (2001). (in Russian). [И.Г. Горячева О.Г. Чекина. Механика дискретного контакта. В сб: Механика контактных взаимодействий. 418-437. М.: Наука. (2001).]

12. V.V. Andronov, V.F. Zhuravlev. Dry friction in mechanical problems. Moscow-Izhevsk. SIC Regular and chaotic dynamics. (2010). 184p. (in Russian). [B.В. Андронов, В.Ф. Журавлев. Сухое трение в задачах механики. М.Ижевск. НИЦ Регулярная и хаотическая динамика. (2010). 184c.]

13. V.V. Andronov. Mechanics of Solids . 1. 40-49. (1988). (in Russian). [В.В. Андронов. Изв.РАН МТT. 1. 40-49. (1988).]

14. K. Johnson. Contact mechanics. Cambridge. Cambridge University Press. (1985). 510 p. 